\title{
Gamma Knife radiosurgery for the treatment of cavernous sinus hemangiomas
}

\author{
QINGSHENG XU*, JIAN SHEN*, YIPING FENG and RENYA ZHAN \\ Department of Neurosurgery, The First Affiliated Hospital, College of Medicine, Zhejiang University, \\ Hangzhou, Zhejiang 310003, P.R. China
}

Received November 24, 2014; Accepted November 26, 2015

DOI: 10.3892/ol.2015.4053

\begin{abstract}
The present retrospective study aimed to analyze the outcome of patients with cavernous sinus hemangioma (CSH) treated with Gamma Knife radiosurgery (GKS). Between August 2011 and April 2014, 7 patients with CSHs underwent GKS. GKS was performed as the sole treatment option in 5 patients, whilst partial resection had been performed previously in 1 patient and biopsy had been performed in 1 patient. The mean volume of the tumors at the time of GKS was $12.5 \pm 10.2 \mathrm{~cm}^{3}$ (range, $5.3-33.2 \mathrm{~cm}^{3}$ ), and the median prescription of peripheral dose was 14.0 Gy (range, 10.0-15.0 Gy). The mean follow-up period was 20 months (range, 6-40 months). At the last follow-up, the lesion volume had decreased in all patients, and all cranial neuropathies observed prior to GKS had improved. There were no radiation-induced neuropathies or complications during the follow-up period. GKS appears to be an effective and safe treatment modality for the management of CSHs.
\end{abstract}

\section{Introduction}

Cavernous sinus hemangiomas (CSHs) are rare extra-axial vascular neoplasms that reportedly account for $<2 \%$ of cavernous sinus tumors, both benign and malignant $(1,2)$. CSHs differ from other intra-axial cavernous malformations in the brain as they represent true vascular neoplasms, and the

Correspondence to: Dr Renya Zhan, Department of Neurosurgery, The First Affiliated Hospital, College of Medicine, Zhejiang University, 79 Qingchun Road, Hangzhou, Zhejiang 310003, P.R. China

E-mail: qsxu10@126.com

*Contributed equally

Abbreviations: CSH, cavernous sinus hemangioma; GKS, Gamma Knife radiosurgery; MRI, magnetic resonance imaging; SRS, stereotactic radiosurgery

Key words: cavernous sinus hemangioma, Gamma Knife, stereotactic radiosurgery associated symptoms are produced as a result of progressive growth of the tumor and mass effects (3).

The optimal treatment strategy for this condition remains controversial. Microsurgical approaches may lead to severe bleeding and even operative death $(4,5)$. As radiotherapy prior to surgical resection has demonstrated some benefit, radiosurgery appears to be a promising treatment modality for the management of lesions in the cavernous sinus. Recently, radiosurgery is being used for primary or postoperative management of patients with CSHs and favorable treatment results have been reported $(1,6)$.

Thus, the current study was conducted with the aim of evaluating the efficacy and safety of Gamma Knife radiosurgery (GKS) in the management of CSHs.

\section{Materials and methods}

Patient characteristics. Between August 2011 and April 2014, 7 patients with CSH who were treated by GKS were enrolled into this retrospective study. With regard to prior treatments, 2 patients had undergone surgical resection prior to GKS, while the remaining 5 patients underwent GKS as a primary treatment under the typical neuroimaging diagnosis including sharply delineated tumors, low to isointense mass lesions on T1-weighted images, extremely high intensity on T2-weighted images (as bright as cerebrospinal fluid signal), and strong homogeneous or heterogeneous enhancement in gadolinium-enhanced imagine, as described elsewhere $(1,7,8)$.

GKS protocol. All treatments were performed using the Leksell Gamma Knife Perfexion ${ }^{\mathrm{TM}}$ (Elekta AB, Stockholm, Sweden). The Leksell Model G stereotactic frame (Elekta AB) was applied to the patient's head under local anesthesia. A high resolution and thin-slice volumetric, gadolinium-enhanced magnetic resonance imaging (MRI) scan was obtained the day prior to treatment. Using the GammaPlan software (Elekta AB, Crawley, UK), the neurosurgeon and medical physicists designed the dose plan. The radiosurgery isodose, maximum dose and marginal dose were initially determined according to the tumor volume calculated during dose planning. Multiple isocenter planning was used to minimize the radiation exposure of the optic pathway and other critical neural structures. 
Table I. Summary of the characteristics of patients with cavernous sinus hemangioma.

\begin{tabular}{|c|c|c|c|c|c|c|c|c|}
\hline \multirow{2}{*}{$\begin{array}{l}\text { Age } \\
\text { (years) }\end{array}$} & \multirow[b]{2}{*}{$\mathrm{M} / \mathrm{F}$} & \multirow{2}{*}{$\begin{array}{l}\text { Presenting } \\
\text { symptom }\end{array}$} & \multirow{2}{*}{$\begin{array}{l}\text { Pre-GKS } \\
\text { treatment }\end{array}$} & \multirow{2}{*}{$\begin{array}{l}\text { Dose } \\
(\mathrm{Gy})\end{array}$} & \multicolumn{2}{|c|}{ Tumor volume $\left(\mathrm{cm}^{3}\right)$} & \multirow{2}{*}{$\begin{array}{l}\text { Follow-up } \\
\text { (months) }\end{array}$} & \multirow{2}{*}{$\begin{array}{l}\text { Volume } \\
\text { reduction }\end{array}$} \\
\hline & & & & & IntraGKS & End & & \\
\hline 45 & $\mathrm{~F}$ & Headache & None & 14 & 10.5 & 5.9 & 6 & MS \\
\hline 59 & M & Diplopia & Surgery & 15 & 3.8 & 1.1 & 12 & $\mathrm{RS}$ \\
\hline 63 & $\mathrm{~F}$ & Facial hypoesthesia & None & 14 & 10.6 & 2.2 & 22 & $\mathrm{RS}$ \\
\hline 41 & $\mathrm{~F}$ & Diplopia & Biopsy & 10 & 33.2 & 2.5 & 40 & $\mathrm{RS}$ \\
\hline 39 & $\mathrm{~F}$ & Headache & None & 12 & 17.5 & 2.0 & 28 & $\mathrm{RS}$ \\
\hline 50 & M & Ophthalmoplegia & None & 15 & 6.5 & 1.2 & 16 & $\mathrm{RS}$ \\
\hline 64 & $\mathrm{~F}$ & Headache & None & 15 & 5.3 & 1.3 & 18 & $\mathrm{RS}$ \\
\hline
\end{tabular}

GKS, Gamma Knife radiosurgery; F, female; M, male; RS, remarkable shrinkage; MS, minimal shrinkage. All patients exhibited an improved symptom response and no complications.
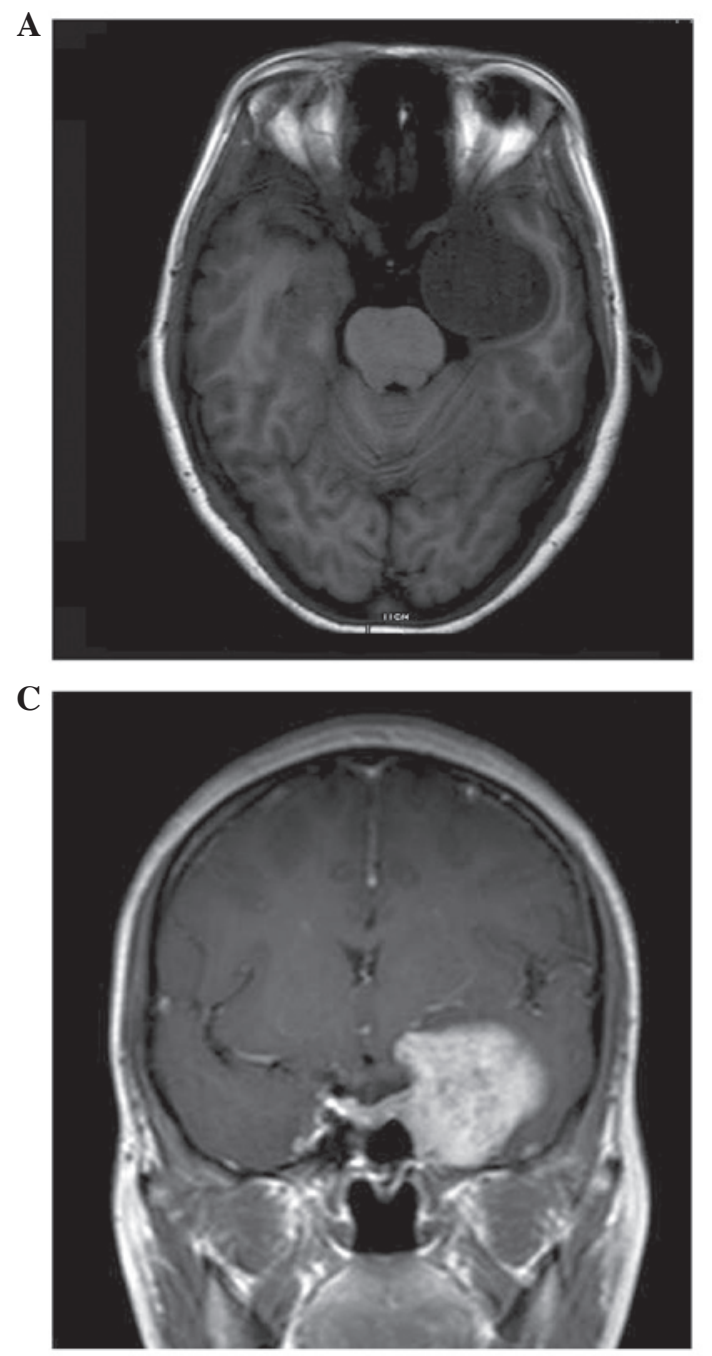
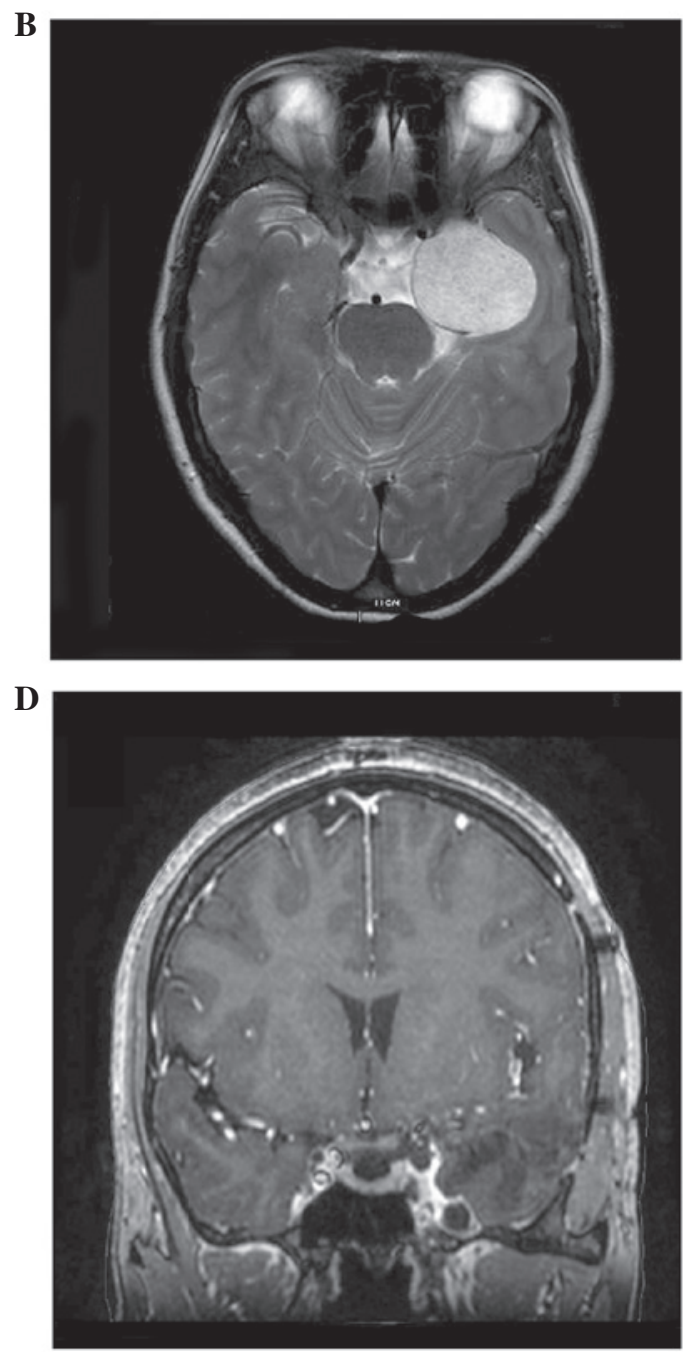

Figure 1. MRI revealed a cavernous sinus hemangioma on the left side. (A) Axial T1-weighted imaging showed a low-signal intensity lesion; (B) T2 weighting revealed a high signal intensity lesion; (C) Coronal T1-weighted contrast-enhanced imaging revealed strong enhancement and a pre-treatment tumor volume of $33.2 \mathrm{~cm}^{3}$, and (D) at 40 months after treatment, the tumor volume shrank to $2.5 \mathrm{~cm}^{3}$.

Follow-up examinations. Following treatment, all patients received regular clinical and radiological follow-up at intervals of 3 months during the first year, and at 6-month intervals thereafter. Tumor control was classified as 'remarkable shrinkage' (tumor volume on follow-up images of $<50 \%$ of the volume prior to GKS), 'minimal shrinkage' (tumor volume of $50-75 \%$ of the tumor volume prior to GKS) or 'stationary' (tumor volume of $75-125 \%$ of the tumor 
volume prior to GKS). 'Tumor progression' covered all other circumstances (9).

\section{Results}

Among the patient group, there were 2 males and 5 females, with a median age of 50.0 years (range, 39-64 years). The predominant symptoms and signs were headache, or symptoms related to diplopia and ophthalmoplegia. The clinical characteristics of the patients and treatment parameters are summarized in Table I.

Radiologically, an iso or low signal intensity on T1-weighted MRI, and a bright, high signal intensity on T2-weighted MRI was observed in all patients. All tumors exhibited strong enhancement following administration of the contrast medium gadolinium-diethylene triamine pentaacetic acid (Fig. 1A-C).

At the time of radiosurgery, the mean volume of the CSHs was $12.5 \pm 10.2 \mathrm{~cm}^{3}$ (range, $5.3-33.2 \mathrm{~cm}^{3}$ ), and the median marginal dose at the $50 \%$ isodose line was 14.0 Gy (range, 10.0-15.0 Gy).

Tumor control was achieved in all patients during the follow-up period. Remarkable shrinkage was achieved in 6 patients and minimal shrinkage was achieved in 1 patient. The patient with minimal shrinkage was observed during the 6 months follow-up, and the volume was reduced from $10.5 \mathrm{~cm}^{3}$ to $5.9 \mathrm{~cm}^{3}$ (reduced $44 \%$ ). In another patient with 40 months of follow-up, the tumor volume reduced from $33.2 \mathrm{~cm}^{3}$ to $2.5 \mathrm{~cm}^{3}$, only $7.5 \%$ of the initial volume. No patients experienced tumor progression prior to the date of last follow-up.

The most common presenting symptom was cranial neuropathy, which affected 4 patients. In these patients, remission of cranial neuropathies was observed following treatment by GKS. Headache was present in 3 patients and was completely resolved following treatment. No adverse radiation effects were observed in the patients during the follow-up period.

\section{Discussion}

Although recent advances in microsurgical techniques have been made, the use of surgery to remove CSHs is frequently challenging (5); this is due to the critical anatomical structures located in the cavernous sinus, and the persistent bleeding from the tumor during surgical resection or even attempted biopsy sampling (6). In a study by Linskey et al (4), in which 52 surgical cases in the literature were reviewed, the total removal rate was only $44 \%$, and cranial neuropathies were exacerbated by surgery in 12 patients $(44.4 \%)$.

For patients with residual or inoperable tumors, radiotherapy often demonstrates benefits in terms of remarkable tumor shrinkage $(10,11)$. Considering the proximity of the optic nerve and optic chiasm, which are considered to be organs at risk of damage during radiotherapy, the use of stereotactic irradiation is increasing (6). Iwai et al (12) reported the first case of CSHs treated with GKS in 1999. Since that time, GKS has been used for primary or postoperative management of patients with CSHs, and favorable treatment results have been reported $(1,6,13)$.
A previous meta-analysis reported that stereotactic radiosurgery (SRS) is an effective and safe treatment option for patients with CSHs; tumor shrinkage was identified in $>90 \%$ of patients, and new cranial neuropathies developed in only $1.7 \%$ of patients (1). In the present study, remarkable post-GKS tumor shrinkage was also identified without causing any new deficits. The long-term results appear to be favorable, and no surgical interventions have been required so far.

The marginal doses delivered to the lesions require the thorough consideration of radiation injury of the critical structures around the cavernous sinus, including the optic apparatus. Nakamura et al (14) suggested that the treatment of CSHs necessitated lower radiosurgical doses compared with those required for other benign tumors. Yamamoto et al (6) analyzed dose-treatment responses in 38 reported cases of CSH treated with radiosurgery, suggesting that a peripheral dose of 14-15 Gy was sufficient for controlling CSH growth, and that 10-12 Gy was the threshold dose for the control of tumor growth. In the current study, 1 patient who was diagnosed by biopsy had a tumor volume of $33.2 \mathrm{~cm}^{3}$ and was treated with a low marginal dose (10 Gy). The tumor volume was subsequently reduced to only $7.5 \%$ of the initial volume, which indicated that $\mathrm{CSH}$ were highly radiosensitive. The ipsilateral abducens nerve palsy was observed to be in a state of partial remission during the 40 months of follow-up (Fig. 1D).

The current study had several limitations, including its small sample size, lack of pathological confirmation and relatively short follow-up period. However, the results may still be helpful considering the prompt response of the CSHs to GKS relatively soon after treatment, and given the rarity of CSHs.

In conclusion, GKS appears to be an effective and safe treatment modality for the management of CSHs, and may be used as a primary treatment in such cases. Further studies with long-term follow-up and larger numbers of cases are necessary to optimize the treatment conditions and verify the benefit of this treatment.

\section{References}

1. Wang X, Mei G, Liu X, Dai J, Pan L and Wang E: The role of stereotactic radiosurgery in cavernous sinus hemangiomas: A systematic review and meta-analysis. J Neurooncol 107: 239-245, 2012.

2. Gliemroth J, Missler U and Sepehrnia A: Cavernous angioma as a rare neuroradiologic finding in the cavernous sinus. J Clin Neurosci 7: 554-557, 2000.

3. Chou CW, Wu HM, Huang CI, Chung WY, Guo WY, Shih YH, Lee LS and Pan DH: Gamma knife surgery for cavernous hemangiomas in the cavernous sinus. Neurosurgery 67: 611-616, 2010.

4. Linskey ME and Sekhar N: Cavernous sinus hemangiomas: A series, a review and an hypothesis. Neurosurgery 30: 101-108, 1992.

5. Zhou LF, Mao Y and Chen L: Diagnosis and surgical treatment of cavernous sinus hemangiomas: An experience of 20 cases. Surg Neurol 60: 31-36, 2003.

6. Yamamoto M, Kida Y, Fukuoka S, Iwai Y, Jokura H, Akabane A and Serizawa T: Gamma Knife radiosurgery for hemangiomas of the cavernous sinus: A seven-institute study in Japan. J Neurosurg 112: 772-777, 2010.

7. Fraser JF, Mass AY, Brown S, Anand VK and Schwartz TH: Transnasal endoscopic resection of a cavernous sinus hemangioma: Technical note and review of the literature. Skull Base 18: 309-315, 2008

8. Kida Y, Kobayashi T and Mori Y: Radiosurgery of cavernous hemangiomas in the cavernous sinus. Surg Neurol 56: 117-123, 2001. 
9. Song SW, Kim DG, Chung HT, Paek SH, Han JH, Kim YH, Kim JW, Kim YH and Jung HW: Stereotactic radiosurgery for cavernous sinus hemangiomas. J Neurooncol 118: $163-168,2014$

10. Rigamonti D, Pappas CT, Spetzler RF and Johnson PC: Extracerebral cavernous angiomas of the middle fossa Neurosurgery 27: 306-310, 1990.

11. Shibata S and Mori K: Effect of radiation therapy on extracerebral cavernous hemangioma in the middle fossa. Report of three cases. J Neurosurg 67: 919-922, 1987.
12. Iwai Y, Yamanaka K, Nakajima H and Yasui T: Stereotactic radiosurgery for cavernous sinus cavernous hemangioma-case report. Neurol Med Chir (Tokyo) 39: 288-290, 1999.

13. Thompson TP, Lunsford LD and Flickinger JC: Radiosurgery for hemangiomas of the cavernous sinus and orbit: Technical case report. Neurosurgery 47: 778-783, 2000.

14. Nakamura N, Shin M, Tago M, Terahara A, Kurita H, Nakagawa K and Ohtomo K: Gamma knife radiosurgery for cavernous hemangiomas in the cavernous sinus. Report of three cases. J Neurosurg 97: 477-480, 2002. 